\title{
Linear programming with Triangular Intuitionistic Fuzzy Number
}

\author{
Dipti Dubey $^{1}$ Aparna Mehra ${ }^{2}$ \\ ${ }^{1}$ Department of Mathematics, Indian Institute of Technology, Hauz Khas, New Delhi-110016, India \\ ${ }^{2}$ Department of Mathematics, Indian Institute of Technology, Hauz Khas, New Delhi-110016, India
}

\begin{abstract}
This paper presents an approach based on value and ambiguity indexes defined in [1] to solve linear programming problems with data as triangular intuitionistic fuzzy numbers.
\end{abstract}

Keywords: Triangular intuitionistic fuzzy number, Fuzzy linear programming, Ranking method.

\section{Introduction}

Decision making problems exhibit some level of imprecisions and vagueness in estimation of model parameters. Such phenomena has been very well captured through fuzzy sets in modeling these problems. Applications of fuzzy set theory in decision making and in particular to optimization problems have been extensively studied ever since the introduction of fuzzy sets by Zadeh [2]. As a result, a large volume of research has appeared in this direction (please see, $[3,4,5])$. The most common concept used in almost all these studies is ranking of fuzzy numbers. Ranking of fuzzy numbers is an important issue in the study of fuzzy set theory. In order to rank fuzzy numbers, one fuzzy number needs to be compared with the others, but it is difficult to determine clearly which of them is larger or smaller. Numerous methods have been proposed in literature to rank fuzzy numbers (for example, please see, $[6,7,8,9,10,11,12]$, and references therein). Also, different methods satisfy different desirable criteria. Many of these methods are based on the area measurement with the integral value about the membership function of fuzzy numbers. Notably, one thing is clear that there exists no uniquely best method for comparing fuzzy numbers.

Recent years have witnessed a growing interest in the study of decision making problems with intuitionistic fuzzy sets/numbers (for example, see, [1, 13, 14, $15,16])$. The intuitionistic fuzzy set (IFS) is an extension of fuzzy set (FS) where the degree of nonmembership denoting the non-belongingness to a set is explicitly specified along with the degree of membership of belongingness to the set. Unlike the FS where the non-membership degree is taken as one minus the membership degree, in IFS, the membership and nonmembership degrees are more or less independent and related only by that the sum of two degrees must not exceed one. Another notable extension of FS is the interval-valued fuzzy set (IVFS) [17], which is characterized by an interval-valued membership function. In [18], Atanassov and Gorgov, and later Deschrijver and Kerre [19] proved that the two concepts of IFS and IVFS are isomorphic to each other. Virtually, they can be used in mathematically equivalent sense.

On the front of ranking intuitionistic fuzzy numbers (IFNs), some work has been reported in the literature $[1,13,20,21,22,23,24]$. Grzegorzewski [20] defined two families of metrics in the space of IFNs and proposed a ranking method for IFNs based on these metrics. Mitchell [21] extended the natural ordering of real numbers to triangular intuitionistic fuzzy numbers (TIFNs) by adopting a statistical view point and interpreting each intuitionistic fuzzy number (IFN) as ensemble of ordinary fuzzy numbers. Li [13] proposed a ranking order relation of TIFN using lexicographic technique. Earlier, Nayagam et al. [22] introduced TIFNs of special type and described a method to rank them. Although their ranking method appears to be attractive, the very definition of TIFN seems unrealistic. This is because the triangular non-membership function is defined to geometrically behave in an identical manner as the membership function. Su [24] investigated the signed distance method for ranking interval valued fuzzy numbers, which for triangular fuzzy numbers becomes analogous to the centroid method. Very recently, Nehi [23] put forward a new ordering method for IFNs in which two characteristic values for IFNs are defined by the integral of the inverse fuzzy membership and non-membership functions multiplied by the grade with powered parameter. Almost parallel, Li [1] introduced a new definition of the TIFN which has an appealing and logically reasonable interpretation. He defined two concepts of the value and the ambiguity of a TIFN similar to those for a fuzzy number introduced by Delgado et al. [25]. These are then used to define the value index and the ambiguity index for TIFN. A ratio ranking method is developed for ordering TIFNs. This double-indexed approach is found to be more robust and effective than any single-index approaches for ranking IFNs. Furthermore, the method also takes into consideration a parameter $\lambda \in[0,1]$ which may reflect the subjective attitude of the decision maker. We shall be elaborating on these aspects in the sections to follow.

In this paper we first defined a TIFN which is more general than the one defined in $[1,13]$. We extend the definitions of the value and the ambiguity index given by Li [1] to the newly defined TIFNs. Our main aim has 
been to research a meaningful approach to handle linear programming problems (LPPs) with data as intuitionistic fuzzy numbers.

The brief description of the paper is as follows. In section 2, besides certain basic definitions, we also present a brief overview of the value and the ambiguity index for TIFNs, and give a new ranking function. In Section 3 , using the new ranking function, a method is proposed to solve linear programming problems with TIFNs. Section 4 presents some illustrative examples. The paper is summarized in Section 5.

\section{Preliminaries}

We quote few a definitions and properties of triangular intuitionistic fuzzy numbers (TIFNs) relevant to the present work.

Definition 1 [26] An intuitionistic fuzzy set (IFS) ã assigns to each element $x$ of the universe $X$ a membership degree $\mu_{\tilde{a}}(x) \in[0,1]$ and a non-membership degree $\nu_{\tilde{a}}(x) \in[0,1]$ such that $\mu_{\tilde{a}}(x)+\nu_{\tilde{a}}(x) \leq 1$. An IFS $\tilde{a}$ is mathematically represented as $\left\{\left\langle x, \mu_{\tilde{a}}(x), \nu_{\tilde{a}}(x)\right\rangle \mid x \in\right.$ $X\}$.

The value $\pi_{\tilde{a}}(x)=1-\mu_{\tilde{a}}(x)-\nu_{\tilde{a}}(x)$ is called the degree of hesitancy or the intuitionistic index of $x$ to $\tilde{a}$.

In this work, $X=\mathbb{R}$. The next few concepts are taken from [1].

Definition 2 A TIFN $\tilde{a}=\left\{\left(\underline{a}^{\mu}, a, \bar{a}^{\mu} ; w_{\tilde{a}}\right)\right.$, $\left.\left(\underline{a}^{\nu}, a, \bar{a}^{\nu} ; u_{\tilde{a}}\right)\right\}$ is an IFS in $\mathbb{R}$, whose membership and non-membership functions are respectively defined as follows:

$$
\begin{gathered}
\mu_{\tilde{a}}(x)= \begin{cases}\frac{\left(x-\underline{a}^{\mu}\right) w_{\tilde{a}}}{a-\underline{a}^{\mu}} & \underline{a}^{\mu} \leq x<a \\
w_{\tilde{a}} & x=a \\
\frac{\left(\bar{a}^{\mu}-x\right) w_{\tilde{a}}}{\bar{a}^{\mu}-a} & a<x \leq \bar{a}^{\mu} \\
0 & \text { otherwise, }\end{cases} \\
\nu_{\tilde{a}}(x)= \begin{cases}\frac{a-x+u_{\tilde{a}}\left(x-\underline{a}^{\nu}\right)}{a-\underline{a}^{\nu}} & \underline{a}^{\nu} \leq x<a \\
u_{\tilde{a}} & x=a \\
\frac{x-a+u_{\tilde{a}}\left(\bar{a}^{\nu}-x\right)}{\bar{a}^{\nu}-a} & a<x \leq \bar{a}^{\nu} \\
1 & \text { otherwise. }\end{cases}
\end{gathered}
$$

The values $w_{\tilde{a}}$ and $u_{\tilde{a}}$ respectively represent the maximum degree of the membership and the nonmembership such that $0 \leq w_{\tilde{a}} \leq 1,0 \leq u_{\tilde{a}} \leq 1$ and $0 \leq w_{\tilde{a}}+u_{\tilde{a}} \leq 1$. The same is depicted in Figure 1.

Observe that the way TIFN is defined here slightly differ from the one defined in [1]. Here, we put forward the idea that $\mu_{\tilde{a}}(x)=0$ does not mean that $\nu_{\tilde{a}}(x)=1$ . Observe that for $x \in\left[\underline{a}^{\nu}, \underline{a}^{\mu}\right]$ and $x \in\left[\bar{a}^{\mu}, \bar{a}^{\nu}\right]$, it is $\mu_{\tilde{a}}(x)=0, \nu_{\tilde{a}}(x)<1$.

In a similar way to the arithmetic operations of the triangular fuzzy numbers (TFNs) and the TIFNs, the arithmetic operations over the above described TIFNs are defined as follows.

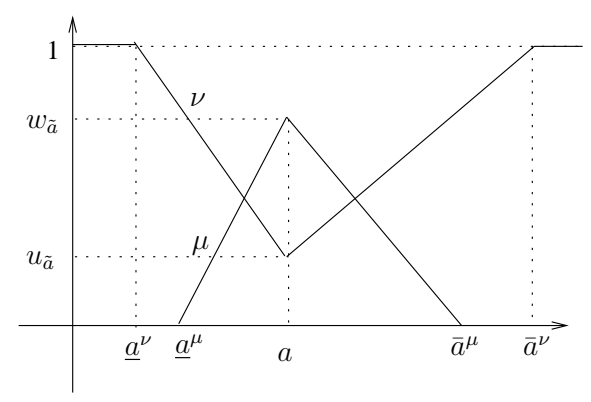

Figure 1: Triangular Intuitionistic Fuzzy number (TIFN)

Definition 3 Let $\tilde{a}=\left\{\left(\underline{a}^{\mu}, a, \bar{a}^{\mu} ; w_{\tilde{a}}\right),\left(\underline{a}^{\nu}, a, \bar{a}^{\nu} ; u_{\tilde{a}}\right)\right\}$ and

$\tilde{b}=\left\{\left(\underline{b}^{\mu}, b, \bar{b}^{\mu} ; w_{\tilde{b}}\right),\left(\underline{b}^{\nu}, b, \bar{b}^{\nu} ; u_{\tilde{b}}\right)\right\}$ be two TIFNs and $k$ be a real number. Then

$$
\begin{aligned}
& \tilde{a}+\tilde{b}=\left\{\left(\underline{a}^{\mu}+\underline{b}^{\mu}, a+b, \bar{a}^{\mu}+\bar{b}^{\mu} ; \min \left\{w_{\tilde{a}}, w_{\tilde{b}}\right\}\right),\right. \\
& \left.\left(\underline{a}^{\nu}+\underline{b}^{\nu}, a+b, \bar{a}^{\nu}+\bar{b}^{\nu} ; \max \left\{u_{\tilde{a}}, u_{\tilde{b}}\right\}\right)\right\} .
\end{aligned}
$$

$k \tilde{a}= \begin{cases}\left\{\left(k \underline{a}^{\mu}, k a, k \bar{a}^{\mu} ; w_{\tilde{a}}\right),\left(k \underline{a}^{\nu}, k a, k \bar{a}^{\nu} ; u_{\tilde{a}}\right)\right\} & k>0 \\ \left\{\left(k \bar{a}^{\mu}, k a, k \underline{a}^{\mu} ; w_{\tilde{a}}\right),\left(k \bar{a}^{\nu}, k a, k \underline{a}^{\nu} ; u_{\tilde{a}}\right)\right\} & k<0 .\end{cases}$

We shall be using value and ambiguity indexes defined in [1]. Following on the lines of $\mathrm{Li}$ [1] we compute the value index and the ambiguity index for the modified TIFNs (Definition 2). We here skip the detailed but straightforward working of how the two indexes have been evolved, and present only the final formulas for them.

Definition 4 Let $\tilde{a}=\left\{\left(\underline{a}^{\mu}, a, \bar{a}^{\mu} ; w_{\tilde{a}}\right),\left(\underline{a}^{\nu}, a, \bar{a}^{\nu} ; u_{\tilde{a}}\right)\right\}$ be a TIFN. Then the value and the ambiguity of $a \tilde{a}$ are given as follows.

(i) The value of the membership function of $\tilde{a}$ is

$$
V_{\mu}(\tilde{a})=\frac{\left(\underline{a}^{\mu}+4 a+\bar{a}^{\mu}\right) w_{\tilde{a}}}{6},
$$

while the value of the non-membership function is

$$
V_{\nu}(\tilde{a})=\frac{\left(\underline{a}^{\nu}+4 a+\bar{a}^{\nu}\right)\left(1-u_{\tilde{a}}\right)}{6} .
$$

(ii) The ambiguity of the membership function of $\tilde{a}$ is

$$
A_{\mu}(\tilde{a})=\frac{\left(\bar{a}^{\mu}-\underline{a}^{\mu}\right) w_{\tilde{a}}}{3},
$$

while the ambiguity of the non-membership function of $\tilde{a}$ is

$$
A_{\nu}(\tilde{a})=\frac{\left(\bar{a}^{\nu}-\underline{a}^{\nu}\right)\left(1-u_{\tilde{a})}\right.}{3} .
$$

Obviously, $A_{\mu}(\tilde{a}) \leq A_{\nu}(\tilde{a})$.

Further, $V_{\mu}(\tilde{a}), V_{\nu}(\tilde{a}), A_{\mu}(\tilde{a})$ and $A_{\nu}(\tilde{a})$ have some useful properties which are summarized below.

\section{Proposition 1 Let}

$\tilde{a}=\left\{\left(\underline{a}^{\mu}, a, \bar{a}^{\mu} ; w_{\tilde{a}}\right),\left(\underline{a}^{\nu}, a, \bar{a}^{\nu} ; u_{\tilde{a}}\right)\right\}$ and $\tilde{b}=$ $\left\{\left(\underline{b}^{\mu}, b, \bar{b}^{\mu} ; w_{\tilde{b}}\right),\left(\underline{b}^{\nu}, b, \bar{b}^{\nu} ; u_{\tilde{b}}\right)\right\}$ be two TIFN's and $k_{1}, k_{2}$ be nonnegative real numbers. Then 
(i) $V_{\mu}\left(k_{1} \tilde{a}+k_{2} \tilde{b}\right)=\min \left\{w_{\tilde{a}}, w_{\tilde{b}}\right\}\left(k_{1} \frac{V_{\mu}(\tilde{a})}{w_{\tilde{a}}}+\right.$ $\left.k_{2} \frac{V_{\mu}(\tilde{b})}{w_{\tilde{b}}}\right)$.

(ii) $V_{\nu}\left(k_{1} \tilde{a}+k_{2} \tilde{b}\right)=\min \left\{1-u_{\tilde{a}}, 1-u_{\tilde{b}}\right\}\left(k_{1} \frac{V_{\nu}(\tilde{a})}{1-u_{\tilde{a}}}\right.$ $\left.k_{2} \frac{V_{\nu}(\tilde{b})}{1-u_{\tilde{b}}}\right)$

(iii) $A_{\mu}\left(k_{1} \tilde{a}+k_{2} \tilde{b}\right)=\min \left\{w_{\tilde{a}}, w_{\tilde{b}}\right\}\left(k_{1} \frac{A_{\mu}(\tilde{a})}{w_{\tilde{a}}}+\right.$ $\left.k_{2} \frac{A_{\mu}(\tilde{b})}{w_{\tilde{b}}}\right)$.

(iv) $A_{\nu}\left(k_{1} \tilde{a}+k_{2} \tilde{b}\right)=\min \left\{1-u_{\tilde{a}}, 1-u_{\tilde{b}}\right\}\left(k_{1} \frac{A_{\nu}(\tilde{a})}{1-u_{\tilde{a}}}\right.$ $\left.k_{2} \frac{A_{\nu}(\tilde{b})}{1-u_{\tilde{b}}}\right)$

Assumption 1 From now onwards we consider those TIFN $\tilde{a}$ that satisfy the condition $V_{\mu}(\tilde{a}) \leq V_{\nu}(\tilde{a})$.

Definition 5 Let $\tilde{a}=\left\{\left(\underline{a}^{\mu}, a, \bar{a}^{\mu} ; w_{\tilde{a}}\right),\left(\underline{a}^{\nu}, a, \bar{a}^{\nu} ; u_{\tilde{a}}\right)\right\}$ be a TIFN. Then the value index and the ambiguity index of $\tilde{a}$ are respectively defined as follows

and

$$
V(\tilde{a}, \lambda)=V_{\mu}(\tilde{a})+\lambda\left(V_{\nu}(\tilde{a})-V_{\mu}(\tilde{a})\right)
$$

$$
A(\tilde{a}, \lambda)=A_{\nu}(\tilde{a})-\lambda\left(A_{\nu}(\tilde{a})-A_{\mu}(\tilde{a})\right),
$$

where $\lambda \in[0,1]$ is a weight which represents the decision maker's (DM) preference information. It allows flexibility to incorporate the subjective attitude of DM in the model. $\lambda \in[0,1 / 2)$ shows the pessimistic behavior while $\lambda \in(1 / 2,1]$ indicates optimistic behavior of the $D M$, and $\lambda=1 / 2$ can be interpreted as an indifferent attitude of the DM.

Note that for any $\lambda, A(\tilde{a}, \lambda) \geq 0$. We say that a TIFN $\tilde{a}$ is non-negative if $V(\tilde{a}, \lambda) \geq 0$. Define

$$
F(\tilde{a}, \lambda)=V(\tilde{a}, \lambda)-A(\tilde{a}, \lambda) .
$$

For a predefined value of $\lambda \in[0,1]$, we define a new ranking (ordering) relation for TIFNs $\tilde{a}$ and $\tilde{b}$ as follows

$$
\tilde{a} \preccurlyeq \tilde{b} \text { if and only if } F(\tilde{a}, \lambda) \leq F(\tilde{b}, \lambda) .
$$

It is reasonable to believe that, more is the value and lesser is the ambiguity of the TIFN the larger is the TIFN. And also, $\lambda>0$ represents the DM's preference information. In view of these facts it makes sense to assume $V_{\mu}(\tilde{a}) \leq V_{\nu}(\tilde{a})$. Thereby the proposed ranking works for a subset of the set of TIFNs which satisfies assumption 1.

Obviously, the ranking order depends on the attitude parameter $\lambda$. It can easily be seen that the proposed ranking function $F$ satisfies properties $A_{1}, A_{2}, A_{3}, A_{5}$ and $A_{6}$ of [11] desired to be satisfied by any reasonable ranking function. For the sake of completeness, we list these properties below.

Let $S$ be the set of fuzzy quantities, and $\mathrm{M}$ be an ordering approach.
$A_{1}$. For an arbitrary finite subset $\mathbb{A}$ of $S$ and $\tilde{a} \in \mathbb{A}$, $\tilde{a} \succcurlyeq \tilde{b}$ by $M$ on $\mathbb{A}$.

$A_{2}$. For an arbitrary finite subset $\mathbb{A}$ of $S$ and $(\tilde{a}, \tilde{b}) \in$ $\mathbb{A}^{2}, \tilde{a} \succcurlyeq \tilde{b}$ and $\tilde{b} \succcurlyeq \tilde{a}$ by $M$ on $\mathbb{A}$, we should have $\tilde{a} \sim \tilde{b}$ by $M$ on $\mathbb{A}$.

$A_{3}$. For an arbitrary finite subset $\mathbb{A}$ of $S$ and $(\tilde{a}, \tilde{b}, \tilde{c}) \in$ $\mathbb{A}^{3} ; \tilde{a} \succcurlyeq \tilde{b}$ and $\tilde{b} \succcurlyeq \tilde{c}$ by $M$ on $\mathbb{A}$, we should have $\tilde{a} \succcurlyeq \tilde{c}$ by $M$ on $\mathbb{A}$.

$A_{4}$. For an arbitrary finite subset $\mathbb{A}$ of $S$ and $(\tilde{a}, \tilde{b}) \in$ $\mathbb{A}^{2}$, inf $\operatorname{supp}(\tilde{a})>\sup \operatorname{supp}(\tilde{b})$, we should have $\tilde{a} \succcurlyeq \tilde{b}$ by $M$ on $\mathbb{A}$.

$A_{5}$. Let $S$ and $S^{\prime}$ be two arbitrary finite sets of fuzzy quantities in which $M$ can be applied and $\tilde{a}$ and $\tilde{b}$ are in $S \cap S^{\prime}$. We obtain the ranking order $\tilde{a} \succ \tilde{b}$ by $M$ on $S^{\prime}$ iff $\tilde{a} \succ \tilde{b}$ by $M$ on $S$.

$A_{6}$. Let $\tilde{a}, \tilde{b}, \tilde{a}+\tilde{c}, \tilde{b}+\tilde{c}$ be elements of $S$. If $\tilde{a} \succcurlyeq \tilde{b}$ by $M$ on $\{\tilde{a}, \tilde{b}\}$, then $\tilde{a}+\tilde{b} \succcurlyeq \tilde{b}+\tilde{c}$ by $M$ on $\{\tilde{a}+\tilde{c}, \tilde{b}+\tilde{c}\}$.

The proposed $F$ does not satisfy $A_{4}$ because for two fuzzy numbers $\tilde{a}$ and $\tilde{b}$ if inf $\operatorname{supp}(\tilde{a})>\sup \operatorname{supp}(\tilde{b})$ then $V(\tilde{a}, \lambda) \geq V(\tilde{b}, \lambda)$ but we can not say anything about $A(\tilde{a}, \lambda)$ and $A(\tilde{b}, \lambda)$. Therefore, there is a possibility that with our ranking approach, $\tilde{a} \preccurlyeq \tilde{b}$. For instance, take two fuzzy numbers $\tilde{a}=(9,10,20 ; 1)$ and $\tilde{b}=(8.7,8.8,8.9 ; 1)$. Then $\inf \operatorname{supp}(\tilde{a})>\sup \operatorname{supp}(\tilde{b})$, but by using the above proposed ranking function $F$ we get $\tilde{a} \preccurlyeq \tilde{b}$.

In continuation, we would like to add that the proposed ranking has an obvious advantage over some other single-index ranking. For instance, if we wish to rank $\tilde{a}=\{(0.2,0.5,0.8 ; 1),(0.2,0.5,0.8 ; 0)\}$ and $\tilde{b}=\{(0.35,0.5,0.65 ; 1),(0.35,0.5,0.65 ; 0)\}$, then they turn out to be equal by any ranking method suggested in [20, 21, 13, 24]. However, using the proposed ranking approach, we can easily say that $\tilde{a} \preccurlyeq \tilde{b}$. It is important to observe the following. For a TIFN $\tilde{a}, V_{\mu}(\tilde{a}) \leq V_{\nu}(\tilde{a})$ by assumption1. Consequently, for $0 \leq \lambda_{1} \leq \lambda_{2} \leq 1$, i.e.,

$$
V\left(\tilde{a}, \lambda_{1}\right)-A\left(\tilde{a}, \lambda_{1}\right) \leq V\left(\tilde{a}, \lambda_{2}\right)-A\left(\tilde{a}, \lambda_{2}\right),
$$

$$
F\left(\tilde{a}, \lambda_{1}\right) \leq F\left(\tilde{a}, \lambda_{2}\right) .
$$

\section{Linear Programming with Triangular Intuitionistic Fuzzy Numbers}

The purpose of this section is to study a class of fuzzy linear programming problems in which the data parameters are TIFNs. Consider the following linear programming problem

$$
\begin{aligned}
& \text { (IFLP) mãx } \quad \sum_{j=1}^{n} \tilde{c}_{j} x_{j} \\
& \text { subject to } \quad \sum_{j=1}^{n} \tilde{a}_{i j} x_{j} \preccurlyeq \tilde{b}_{i}, i=1, \ldots, m \\
& \quad x_{j} \geq 0, j=1, \ldots, n,
\end{aligned}
$$

where, $\tilde{c}_{j}=\left\{\left(\underline{c}_{j}^{\mu}, c_{j}, \bar{c}_{j}^{\mu} ; w_{\tilde{c}_{j}}\right),\left(\underline{c}_{j}^{\nu}, c_{j}, \bar{c}_{j}^{\nu} ; u_{\tilde{c}_{j}}\right)\right\}$, $\tilde{b}_{i}=\left\{\left(\underline{b}_{i}^{\mu}, b_{i}, \bar{b}_{j}^{\mu} ; w_{\tilde{b}_{i}}\right),\left(\underline{b}_{i}^{\nu}, b_{i}, \bar{b}_{i}^{\nu} ; u_{\tilde{b}_{i}}\right)\right\}$ and $\tilde{a}_{i j}=\left\{\left(\underline{a}_{i j}^{\mu}, a_{i j}, \bar{a}_{i j}^{\mu} ; w_{\tilde{a}_{i j}}\right),\left(\underline{a}_{i j}^{\nu}, a_{i j}, \bar{a}_{i j}^{\nu} ; u_{\tilde{a}_{i j}}\right)\right\}$, $i=1, \ldots, m, j=1, \ldots, n$, are TIFN's. 
Using the ranking function $F$, for a predefined $\lambda \in$ $[0,1]$, (IFLP) is equivalent to the following crisp optimization problem.

$$
\begin{aligned}
(\mathrm{COP})_{\lambda} \max & F\left(\sum_{j=1}^{n} \tilde{c}_{j} x_{j}, \lambda\right) \\
\text { subject to } F\left(\sum_{j=1}^{n} \tilde{a}_{i j} x_{j}, \lambda\right) & \leq F\left(\tilde{b}_{i}, \lambda\right), i=1, \ldots, m \\
x_{j} & \geq 0, j=1, \ldots, n .
\end{aligned}
$$

Further, using (1) and Proposition 1, problem $(\mathrm{COP})_{\lambda}$ is equivalent to the following linear programming problem.

subject to

$$
\begin{aligned}
& \max (1-\lambda) \min _{j}\left\{w_{\tilde{c}_{j}}\right\} \sum_{j=1}^{n} \frac{V_{\mu}\left(\tilde{c}_{j}\right)}{w_{\tilde{c}_{j}}} x_{j} \\
& \left.-(1-\lambda) \min _{j}\left\{1-u_{\tilde{c}_{j}}\right\} \sum_{j=1}^{n} \frac{A_{\nu}\left(\tilde{c}_{j}\right)}{1-u_{\tilde{c}_{j}}}\right) x_{j} \\
& +\lambda \min _{j}\left\{1-u_{\tilde{c}_{j}}\right\} \sum_{j=1}^{n} \frac{V_{\nu}\left(\tilde{c}_{j}\right)}{1-u_{\tilde{c}_{j}}} x_{j} \\
& -\lambda \min _{j}\left\{w_{\tilde{c}_{j}}\right\} \sum_{j=1}^{n} \frac{A_{\mu}\left(\tilde{c}_{j}\right)}{w_{\tilde{c}_{j}}} x_{j}
\end{aligned}
$$

$$
\begin{aligned}
& (1-\lambda) \min _{i}\left\{w_{\tilde{a}_{i j}}\right\} \sum_{j=1}^{n} \frac{V_{\mu}\left(\tilde{a}_{i j}\right)}{w_{\tilde{a}_{i j}}} x_{j} \\
& -(1-\lambda) \min _{i}\left\{1-u_{\tilde{a}_{i j}}\right\} \sum_{j=1}^{n} \frac{A_{\nu}\left(\tilde{a}_{i j}\right)}{1-u_{\tilde{a}_{i j}}} x_{j} \\
& +\lambda \min _{i}\left\{1-u_{\tilde{a}_{i j}}\right\} \sum_{j=1}^{n} \frac{V_{\nu}\left(\tilde{a}_{i j}\right)}{1-u_{\tilde{a}_{i j}}} x_{j} \\
& -\lambda \min _{i}\left\{w_{\tilde{a}_{i j}}\right\} \sum_{j=1}^{n} \frac{A_{\mu}\left(\tilde{a}_{i j}\right)}{w_{\tilde{a}_{i j}}} x_{j} \leq \\
& (1-\lambda)\left(V_{\mu}\left(b_{i}\right)-A_{\nu}\left(b_{i}\right)\right)+\lambda\left(V_{\nu}\left(b_{i}\right)-A_{\mu}\left(b_{i}\right)\right) \\
& x_{j} \geq 0 \quad j=1, \ldots, \ldots
\end{aligned}
$$

Here we assume that the DM is rational enough to provide the intuitionistic fuzzy data such that problem $(\mathrm{CLP})_{\lambda}$ remains bounded and feasible for at least one choice of $\lambda$.

For $\lambda=1,(\mathrm{CLP})_{\lambda}$ reduce to

$$
\begin{aligned}
\max \min _{j}\left\{1-u_{\tilde{c}_{j}}\right\} & \sum_{j=1}^{n} \frac{V_{\nu}\left(\tilde{c}_{j}\right)}{1-u_{\tilde{c}_{j}}} x_{j} \\
& -\min _{j}\left\{w_{\tilde{c}_{j}}\right\} \sum_{j=1}^{n} \frac{A_{\mu}\left(\tilde{c}_{j}\right)}{w_{\tilde{c}_{j}}} x_{j}
\end{aligned}
$$

subject to

$$
\begin{gathered}
\min _{i}\left\{1-u_{\tilde{a}_{i j}}\right\} \sum_{j=1}^{n} \frac{V_{\nu}\left(\tilde{a}_{i j}\right)}{1-u_{\tilde{a}_{i j}}} x_{j} \\
-\min _{i}\left\{w_{\tilde{a}_{i j}}\right\} \sum_{j=1}^{n} \frac{A_{\mu}\left(\tilde{a}_{i j}\right)}{w_{\tilde{a}_{i j}}} x_{j} \leq\left(V_{\nu}\left(b_{i}\right)-A_{\mu}\left(b_{i}\right)\right), \\
x_{j} \geq 0, j=1, \ldots, n .
\end{gathered}
$$

If we take $\lambda=0$ in $(\mathrm{CLP})_{\lambda}$, we get the following optimization problem

$$
\begin{aligned}
\max \min _{j}\left\{w_{\tilde{c}_{j}}\right\} \sum_{j=1}^{n} & \frac{V_{\mu}\left(\tilde{c}_{j}\right)}{w_{\tilde{c}_{j}}} x_{j} \\
& \left.\quad-\min _{j}\left\{1-u_{\tilde{c}_{j}}\right\} \sum_{j=1}^{n} \frac{A_{\nu}\left(\tilde{c}_{j}\right)}{1-u_{\tilde{c}_{j}}}\right) x_{j}
\end{aligned}
$$

subject to

$$
\begin{gathered}
\min _{i}\left\{w_{\tilde{a}_{i j}}\right\} \sum_{j=1}^{n} \frac{V_{\mu}\left(\tilde{a}_{i j}\right)}{w_{\tilde{a}_{i j}}} x_{j} \\
-\min _{i}\left\{1-u_{\tilde{a}_{i j}}\right\} \sum_{j=1}^{n} \frac{A_{\nu}\left(\tilde{a}_{i j}\right)}{1-u_{\tilde{a}_{i j}}} x_{j} \leq\left(V_{\nu}\left(b_{i}\right)-A_{\mu}\left(b_{i}\right)\right) \\
x_{j} \geq 0, j=1, \ldots, n .
\end{gathered}
$$

For the sake of observation, consider a particular situation when only $c_{j}, j=1, \ldots, n$, are TIFNs and the rest of the data parameters are crisp numbers in a linear program.

$$
\begin{aligned}
& \text { (IFOBLP) } \max \quad \sum_{j=0}^{n} \tilde{c}_{j} x_{j} \\
& \text { subject to } \sum_{j=1}^{n} a_{i j} x_{j} \leq b_{i}, \quad i=1, \ldots, m \\
& x_{j} \geq 0, j=1, \ldots, n .
\end{aligned}
$$

Using the proposed ranking function, (IFOBLP) is equivalent to the following crisp problem.

$$
\begin{aligned}
& (\mathrm{COBLP})_{\lambda} \quad \max F\left(\sum_{j=1}^{n} \tilde{c}_{j} x_{j}, \lambda\right) \\
& \text { subject to } \quad \sum_{j=1}^{n} a_{i j} x_{j} \leq b_{i}, i=1, \ldots, m \\
& x_{j} \geq 0, j=1, \ldots, n .
\end{aligned}
$$

It is worth to notice that if $x_{0}^{*}, x_{\lambda_{1}}^{*}$ and $x_{1}^{*}$ are optimal solutions of $(\mathrm{COLP})_{0},(\mathrm{COLP})_{\lambda_{1}}, 0<\lambda_{1}<1$, and $(\mathrm{COLP})_{1}$, respectively. Then

$F\left(\sum_{j=1}^{n} \tilde{c}_{j} x_{0 j}^{*}, 0\right)<F\left(\sum_{j=1}^{n} \tilde{c}_{j} x_{\lambda_{1} j}^{*}, \lambda_{1}\right)<F\left(\sum_{j=1}^{n} \tilde{c}_{j} x_{1 j}^{*}, 1\right)$.

In other words, the optimal objective value will increasing when the DM gradually moves from pessimistic attitude $(\lambda=0)$ to optimistic attitude $(\lambda=1)$. Thus, the ranking ordering is consistent with our common sense of decision making that, if the feasible set of the problem remains the same then, the optimistic viewpoint should always yield a better objective value than the one we get with pessimistic thinking.

\section{Numerical Illustration}

We present few examples to depict the working of the proposed ranking technique for linear programming problem wherein the data is specified as satisfying assumption 1 .

Example 1 Consider the following intuitionistic fuzzy linear program 


$$
\begin{array}{cl}
\max & \tilde{5} x_{1}+\tilde{3} x_{2} \\
\text { subject to } & \tilde{4} x_{1}+\tilde{3} x_{2} \preccurlyeq \tilde{12} \\
& \tilde{1} x_{1}+\tilde{3} x_{2} \preccurlyeq \tilde{6} \\
& x_{1}, x_{2} \geq 0,
\end{array}
$$

where

$$
\begin{aligned}
c_{1}=\tilde{5} & =\left\{\left(4,5,6 ; \frac{3}{4}\right),\left(4,5,6.1 ; \frac{1}{4}\right)\right\}, \\
c_{2}=\tilde{3} & =\left\{\left(2.5,3,3.2 ; \frac{1}{2}\right),\left(2,3,3.5 ; \frac{1}{4}\right)\right\}, \\
a_{11}=\tilde{4} & =\{(3.5,4,4.1 ; 1),(3,4,5 ; 0)\}, \\
a_{12}=\tilde{3} & =\left\{\left(2.5,3,3.5 ; \frac{3}{4}\right),\left(2.4,3,3.6 ; \frac{1}{5}\right)\right\}, \\
a_{21}=\tilde{1} & =\{(0,1,2 ; 1),(0,1,2 ; 0)\}, \\
a_{22}=\tilde{3} & =\left\{\left(2.8,3,3.2 ; \frac{3}{4}\right),\left(2.5,3,3.2 ; \frac{1}{6}\right)\right\}, \\
b_{1}=\tilde{1} 2 & =\{(11,12,13 ; 1),(11,12,14 ; 0)\}, \\
b_{2}=\tilde{6} & =\left\{\left(5.5,6,7.5 ; \frac{3}{4}\right),\left(5,6,8.1 ; \frac{1}{4}\right)\right\} .
\end{aligned}
$$

For $\lambda=1$, using the method described in earlier section, the equivalent crisp formulation is

$$
\begin{aligned}
& \max \quad 3.0125 x_{1}+1.8209 x_{2} \\
& \text { subject to } \quad 3.05 x_{1}+2.15 x_{2} \leq 11.8327 \\
& 0.3333 x_{1}+4.8166 x_{2} \leq 8.75 \\
& x_{1}, x_{2} \geq 0 \text {. }
\end{aligned}
$$

The optimal solution of the problem is $x_{1}^{*}=$ $3.8795, x_{2}^{*}=0$ with optimal objective value 11.6872 .

We next solve the given program for $\lambda=0$. Following the directions specified in earlier section, we formulate the equivalent crisp model as follows.

$$
\begin{array}{rrl}
\max & 1.975 x_{1}+1.1 x_{2} & \\
\text { subject to } & 2.4165 x_{1}+1.93 x_{2} & \leq 11.25 \\
& 0.1944 x_{1}+2.055 x_{2} & \leq 3.875 \\
x_{1}, x_{2} & \geq 0 .
\end{array}
$$

The optimal solution is $x_{1}^{*}=4.6554, x_{2}^{*}=0$, with optimal objective value 9.1946 .

Next we apply our technique to the problems considered by $\mathrm{Su}$ [24]. The first problem is the one where only the objective function coefficients are taken as IFNs.

\section{Example 2 Consider the intuitionistic linear program}

$$
\begin{aligned}
& \max \quad \tilde{25} x_{1}+\tilde{48} x_{2} \\
& \text { subject to } 15 x_{1}+30 x_{2} \leq 45000 \\
& 24 x_{1}+6 x_{2} \leq 24000 \\
& 21 x_{1}+14 x_{2} \leq 28000 \\
& x_{1}, x_{2} \geq 0 \text {. }
\end{aligned}
$$

where

$$
\begin{aligned}
& c_{1}=\tilde{25}=\{(19,25,33 ; 0.9),(18,25,34 ; 1)\} \\
& c_{2}=\tilde{48}=\{(44,48,54 ; 0.9),(43,48,56 ; 1)\}
\end{aligned}
$$

Applying the ranking function $F$, the corresponding crisp linear programs for $\lambda=1$ and $\lambda=0$ are respectively given as follows.

$$
\begin{array}{rr}
\max & 21.1333 x_{1}+47 x_{2} \\
\text { subject to } & 15 x_{1}+30 x_{2} \leq 45000 \\
24 x_{1}+6 x_{2} & \leq 24000 \\
21 x_{1}+14 x_{2} & \leq 28000 \\
x_{1}, x_{2} & \geq 0 .
\end{array}
$$

The optimal solution is $x_{1}^{*}=0, x_{2}^{*}=1500$, with optimal objective value 70500 .

$$
\begin{array}{rr}
\max & 17.467 x_{1}+41.33 x_{2} \\
15 x_{1}+30 x_{2} & \leq 45000 \\
24 x_{1}+6 x_{2} & \leq 24000 \\
21 x_{1}+14 x_{2} & \leq 28000 \\
x_{1}, x_{2} & \geq 0 .
\end{array}
$$

The optimal solution is $x_{1}^{*}=0, x_{2}^{*}=1500$, with optimal objective value 62000.10 .

Remark 1 With the increase in the ambiguity of IFNs representing the cost coefficients, we observe that the values of the cost coefficients in the aforementioned crisp formulation reduce while, in the analogous formulation in [24], they increase. Due to this, the optimal value obtained by Su [24] is higher than the one obtained herein for both cases.

The other example in $\mathrm{Su}$ [24] has been of linear program in which the objective function coefficients are crisp numbers while the technology and resource coefficients are TIFNs.

\section{Example 3 Consider the following problem}

$$
\begin{aligned}
& \max 25 x_{1}+48 x_{2} \\
& \text { subject to } \quad \tilde{15} x_{1}+\tilde{30} x_{2} \preccurlyeq 45 \tilde{0} 00 \\
& \tilde{24} x_{1}+\tilde{6} x_{2} \preccurlyeq 24000 \\
& \tilde{21} x_{1}+\tilde{14} x_{2} \preccurlyeq 28000 \\
& x_{1}, x_{2} \geq 0 \text {, }
\end{aligned}
$$

$$
\begin{aligned}
& a_{11}=\tilde{15}=\{(14,15,17 ; 0.9),(10,15,18 ; 0\}, \\
& a_{12}=\tilde{30}=\{(25,30,34 ; 0.9),(23,30,38 ; 0)\}, \\
& a_{21}=\tilde{24}=\{(21,24,26 ; 0.9),(20,24,33 ; 0)\}, \\
& a_{22}=\tilde{6}=\{(4,6,8 ; 0.9),(2,6,11 ; 0)\}, \\
& a_{31}=\tilde{17}=\{(17,21,22 ; 0.9),(16,21,26 ; 0)\}, \\
& a_{32}=\tilde{14}=\{(12,14,19 ; 0.9),(8,14,22 ; 0)\}, \\
& b_{1}=45 \tilde{0} 00=\{(44980,45000,45030 ; 0.9), \\
&(44970,45000,45070 ; 0)\}, \\
& b_{2}=24 \tilde{0} 00 \quad=\{(23980,24000,24050 ; 0.9), \\
&(23940,24000,24060 ; 0)\} \\
& b_{3}=28 \tilde{0} 00=\{(27990,28000,28030 ; 0.9), \\
&(27950,28000,28040 ; 0\}
\end{aligned}
$$

Using the approach of the paper, the associated crisp linear programs for $\lambda=1$ and $\lambda=0$ are respectively as follows.

$$
\begin{aligned}
& \max \quad 25 x_{1}+48 x_{2} \\
& \text { subject to } 13.7666 x_{1}+27.4666 x_{2} \leq 44991.6667 \\
& 23.3333 x_{1}+5.7 x_{2} \leq 23979 \\
& 19.5 x_{1}+12.2333 x_{2} \leq 27986.333 \\
& x_{1}, x_{2} \geq 0 \text {. }
\end{aligned}
$$

The optimal solution is $x_{1}^{*}=594.4993, x_{2}^{*}=1340.08$, with optimal objective value 79186.31 .

$$
\begin{array}{rrl}
\max & 25 x_{1}+48 x_{2} & \\
\text { subject to } & 10.9834 x_{1}+21.85 x_{2} & \leq 44968.333 \\
18.45 x_{1}+4.5 x_{2} & \leq 21564.5 \\
& 15.1167 x_{1}+7.717 x_{2} & \leq 25191 \\
x_{1}, x_{2} & \geq 0 .
\end{array}
$$

The optimal solution is $x_{1}^{*}=760.0264, \quad x_{2}^{*}=$ 1676.003, with optimal objective value 99448.79. 
Remark 2 In the above example, it can easily be checked that the feasible set of the crisp optimization problem in [24] is contained in the feasible set of the above described crisp model for $\lambda=1$, which is further contained in the feasible set described by the crisp model for $\lambda=0$. This leads to an improvement in the optimal values of the objective function in our model of the example as compared to that of Su [24].

However, in general, we can not make any comparative statement between the crisp model of an intuitionistic fuzzy linear programming problem proposed in this paper and the one proposed by Su [24].

\section{Concluding Remarks}

We have defined a more general definition of TIFN than the ones existing in literature. The value and the ambiguity indexes defined by [1] have been computed for these TIFNs. Thereafter, a ranking function has been proposed keeping central thought that the same has to be used to solve a class of linear programming problems in which the data parameters are TIFNs. The solution methodology for such a class of linear programs is illustrated through examples. In this paper we have considered the TIFNs which satisfy assumption 1 . Indeed this is a limitation of the present work.

The task of developing a more effective ranking method for a broader class of TIFNs which can also be effectively applied to solve linear programming problems with intuitionistic fuzzy parameters is still an open research issue.

Acknowledgements: The first author would like to thank the National Board of Higher Mathematics (NBHM), India, for financial support for research. The authors are indebted to the referees for their valuable suggestions and observations.

\section{References}

[1] D. F. Li. A ratio ranking method of triangular intuitionistic fuzzy numbers and its application to madm problems. Computer and Mathematics with Applications, 60:1557-1570, 2010.

[2] Lotfi A. Zadeh. Fuzzy sets. Information and Control, 8:338-353, 1965.

[3] R. E. Bellman and L. A. Zadeh. Decision making in fuzzy environment. Management Sciences, 17:B-141-B-164, 1970.

[4] C. R. Bector and S. Chandra. Fuzzy Mathematical Programming and Fuzzy Matrix Games. Springer Verlag, 2005.

[5] M. Inuiguchi, H. Ichihashi, and Y. Kume. Relationships between modality constrained programming problems and various fuzzy mathematical programming problems. Fuzzy Sets and Systems, 49:243-259, 1992.

[6] S. Abbasbandy. Ranking of fuzzy numbers, some recent and new formulas. In IFSA-EUSFLAT 2009 , pages 642-646, 2009.
[7] T. C. Chu and C. T. Tsao. Ranking fuzzy numbers with an area between the centroid point and original point. Computers Mathematics and Applications, 43:111-117, 2002.

[8] M. Jimenez. Ranking fuzzy numbers through the comparison of its expected intervals. International Journal of Uncertainty, Fuzziness and KnowledgeBased Systems, 4:379-388, 1996.

[9] X. W. Liu and S. L. Han. Ranking fuzzy numbers with preference weighting function expectations. Computers and Mathematics with Applications, 49:1731-1753, 2005.

[10] H.R. Maleki. Ranking functions and their applications to fuzzy linear programming. Far East Journal of Mathematical Sciences, 4:283-301, 2002.

[11] X. Wang and E. E. Kerre. Reasonable properties for the ordering of fuzzy quantities (i). Fuzzy Sets and Systems, 118:375-385, 2001.

[12] X. Wang and E. E. Kerre. Reasonable properties for the ordering of fuzzy quantities (ii). Fuzzy Sets and Systems, 118:387-405, 2001.

[13] J. X. Nan and D.-F. Li. A lexicographic method for matrix games with payoffs of triangular intuitionistic fuzzy numbers. International Journal of Computational Intelligence Systems, 3:280-289, 2010.

[14] D. F. Li. Multiattribute decision making models and methods using intuitionistic fuzzy sets. Journal of Computer and System Sciences, 70:73-85, 2005.

[15] D. F. Li. Extension of the linmap for multiattribute decision making under atanassov's intuitionistic fuzzy environment. Fuzzy Optimization and Decision Making, 7:17-34, 2008.

[16] D. F. Li, G. H. Chen, and Z. G. Huang. Linear programming method for multiattribute decision making using if sets. Information Sciences, 180:15911609, 2010.

[17] Lotfi A. Zadeh. The concept of a linguistic variable and its application to approximate reasoning. Information Sciences, 8:199-249, 1975.

[18] K. T. Atanassov. Interval valued intuitionistic fuzzy sets. Fuzzy Sets and Systems, 31:343-349, 1989.

[19] G. Deschrijver and E. E. Kerre. On the relationship between some extensions of fuzzy set theory. Fuzzy Sets and Systems, 133:227-235, 2003.

[20] P. Grzegorzewski. Distances and orderings in a family of intuitionistic fuzzy numbers. In Proceedings of the Third Conference on Fuzzy Logic and Technology (Eusflat03), pages 223-227, 2003.

[21] H. B. Mitchell. Ranking intuitionistic fuzzy numbers. International Journal of Uncertainity, Fuzziness and Knowledge-Based Systems, 12:377-386, 2004.

[22] V. L. Nayagam, G. Vankateshwari, and G. Sivaraman. Ranking of intuitionistic fuzzy numbers. In 2008 IEEE International Conference on Fuzzy Systems, pages 1971-1974, 2008.

[23] H. M. Nehi. A new ranking method for intuitionistic fuzzy numbers. International Journal of Fuzzy 
Systems, 12:80-86, 2010.

[24] J.-S. Su. Fuzzy programming based on intervalvalued fuzzy numbers and ranking. International Journal of Contempraroy Mathematical Sciences, 2:393-410, 2007.

[25] M. Delgado, M. A. Vila, and W. Voxman. On a canonical representation of fuzzy numbers. Fuzzy Sets and Systems, 93:125-135, 1998.

[26] K.T. Atanassov. Intutionistic fuzzy sets. Fuzzy Sets and Systems, 20:87-96, 1986. 